\title{
A "Tea and Cookies" Approach: Co-designing Cancer Screening Interventions with Patients Living with Low Income
}

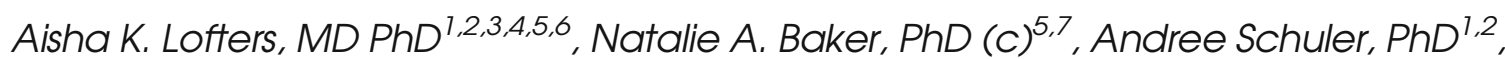 \\ Allison Rau, MSc ${ }^{7}$, Alison Baxter, $\mathrm{MA}^{7}$, Nancy N. Baxter, MD ${ }^{4,5,8}$, Edward Kucharski, MD', \\ Fok-Han Leung, $M D^{2,3}$, Karen Weyman, $M D^{2,3}$, and Tara Kiran, $M D^{1,2,3,4,10,11}$
}

'MAP Centre for Urban Health Solutions, Li Ka Shing Knowledge Institute, St. Michael's Hospital ,Toronto, ON , Canada; ${ }^{2}$ Department of Family and Community Medicine, St. Michael's Hospital , Toronto , ON ,Canada; ${ }^{3}$ Department of Family and Community Medicine, University of Toronto , Toronto, ON , Canada; ${ }^{4} \mathrm{CEES}$, Toronto, ON , Canada; ${ }^{5}$ Dalla Lana School of Public Health , Toronto , Ontario , Canada; ${ }^{6}$ Women's College Hospital , Toronto , ON ,Canada; ${ }^{7}$ Applied Health Research Centre, Li Ka Shing Knowledge Institute, St. Michael's Hospital , Toronto , ON , Canada; ${ }^{8}$ Department of Surgery, Li Ka Shing Knowledge Institute, St. Michael's Hospital , Toronto , ON , Canada; ${ }^{9}$ Cancer Care Ontario , Toronto , ON , Canada; ${ }^{10}$ Health Quality Ontario ,Toronto, ON ,Canada; ${ }^{11}$ Institute of Health Policy, Management and Evaluation , Toronto , ON , Canada.

BACKGROUND: In our primary care organization, we have observed income gradients in cancer screening for our patients despite outreach. We hypothesized that outreach strategies could be improved upon to be more compelling for our patients living with low income.

OBJECTIVE: To use co-design to adapt our current strategies and create new strategies to improve cancer screening uptake for patients living with low income.

DESIGN: An exploratory, qualitative study in two phases: interviews and focus groups.

PARTICIPANTS: For interviews, we recruited 25 patient participants who were or had been overdue for cancer screening and had been identified by their provider as potentially living with low income. For subsequent focus groups, we recruited 14 patient participants, 11 of whom had participated in Phase I interviews.

APPROACH: To analyse written transcripts, we took an iterative, inductive approach using content analysis and drawing on best practices in Grounded Theory methodology. Emergent themes were expanded and clarified to create a derived model of possible strategies to improve the experience of cancer screening and encourage screening uptake for patients living with low income.

KEY RESULTS: Fear and competing priorities were two key barriers to cancer screening identified by patients. Patients believed that a warm and encouraging outreach approach would work best to increase cancer screening participation. Phone calls and group education were specifically suggested as potentially promising methods. However, these views were not universal; for example, women were more likely to be in favour of group education.

CONCLUSIONS: We used input from patients living with low income to co-design a new approach to cancer screening in our primary care organization, an approach that could be broadly applicable to other contexts and settings. We learned from our patients that a multi-modal strategy will likely be best to maximize screening uptake.

Received February 12, 2019

Revised August 16, 2019

Accepted September 3, 2019

Published online October 21, 2019
J Gen Intern Med 35(1):255-60

DOI: $10.1007 / \mathrm{s} 11606-019-05400-0$

(c) Society of General Internal Medicine 2019

\section{BACKGROUND}

Screening for breast, cervical and colorectal cancers are supported by evidence as methods of reducing cancer morbidity and mortality, and are commonly promoted, conducted and/or arranged by primary care providers ${ }^{1}$. In the Canadian province of Ontario, organized screening programmes exist for each of these three cancers with clear eligibility guidelines and organized invitation, reminder and recall methods ${ }^{2-5}$. Despite these efforts, screening rates in Ontario are currently below provincial targets and there are well-documented income-related disparities in screening for all three cancer types ${ }^{6-12}$. Women living in Ontario's lowest income neighbourhoods have been found to have cervical screening rates of $54.3 \%$ versus $66.7 \%$ for women living in the highest income neighbourhoods, and $49.7 \%$ of people living in the lowest income neighbourhoods are overdue for colorectal cancer screening versus $34.9 \%$ of people in the highest income neighbourhoods ${ }^{13}$. Similarly, Vahabi et al. found that $58 \%$ of women living in Ontario's lowest income neighbourhoods versus $69 \%$ of women in the highest income neighbourhoods were up to date on breast cancer screening ${ }^{14}$.

In our primary care organization of over 46,000 patients in Toronto, Ontario's largest city, we have similarly observed income-based cancer screening gradients for our patients 15 , 16 . For example, among our patients whose self-reported income was below the low-income threshold defined by Statistics Canada ${ }^{17}$, only $68.6 \%$ were up-to-date on colorectal cancer screening versus $74.9 \%$ of patients whose income was above the threshold ${ }^{15}$. We have conducted several quality improvement initiatives aimed at both increasing our overall screening rates and reducing the income gradient, including using both reminder letters signed by the primary care physician and reminder phone calls for patients overdue for 
screening. Phone calls were more effective than letters, in that $41.2 \%$ of women and $28.8 \%$ of men allocated to receive a phone call received at least one screening test for which they were due, versus $33.0 \%$ of women and $24.8 \%$ of men allocated to receive a letter. However, they were also more expensive, and neither reminder method led to a significant decrease in the income gradient ${ }^{18}$. We hypothesized that both outreach strategies could be improved upon to be more compelling for our patients living with low income. We also recognized that input from patients could lead us to strategies that we may not yet have considered. Co-design refers to the integration of knowledge of professionals and consumers in service design 19,20 . Thus, the objective of this qualitative study was to use co-design to adapt our current strategies and create new strategies to improve cancer screening uptake for patients living with low income.

\section{METHODS}

\section{Overview}

We used an exploratory, qualitative approach, guided by a community-based participatory and grounded theoretical lens, in two phases ${ }^{21-23}$. Phase I consisted of twenty-five in-depth, semi-structured individual interviews held in late 2017, which were conducted with both women and men to explore their experiences and views of cancer screening. Phase II consisted of three focus groups held in January to March of 2018, conducted to better understand the emerging results from Phase I. One focus group consisted of females only (four participants), one focus group consisted of males only (seven participants) and the final group consisted of three participants: two male, one female.

To guide the research, the team assembled an advisory committee composed of clinicians, researchers, patients, and a representative from the provincial cancer agency. In keeping with our participatory lens, the advisory committee met approximately quarterly to provide input throughout the research process, review findings of each phase, and suggest adaptations as needed ${ }^{21}$.

Ethics approval for the study was provided by the St. Michael's Hospital Research Ethics Board.

\section{Phase I: Individual Interviews}

Sample Selection and Recruitment. We recruited patients for individual interviews using both convenience and purposeful sampling. We gave 21 primary care providers who were members of either the organization's Cancer Screening Working Group or Social Determinants of Health Committee (and thus likely to be engaged in the topic) a list of their patients who were at least six months overdue for cervical, breast, or colorectal cancer screening. We asked them to identify $10-15$ patients from their practices who might be eligible for the study. Eligible patients were defined as:
1. overdue for cervical, breast, or colorectal cancer screening for at least six months as of March 31, 2016

2. comfortable conversing in English, and

3. perceived by their provider to be living with a low income.

We then sent a personalized invitation letter from the provider to identified patients. Letters were followed up with a phone call 1-4 weeks later, unless the patient had already called to state they were or were not interested in participation.

Data Collection. The semi-structured interview guide was developed by the research team and was informed by a desired focus on solutions that could be implemented by our primary care organization. Participants were asked about their level of awareness and personal history of cancer screening in order to understand their personal experiences, barriers and facilitators to screening that would need to be considered for screening interventions, and their perspectives on potential solutions for improving cancer screening uptake for people living with low income. Participants were also asked to provide feedback on the outreach strategies ( letters and phone calls) that the organization has used in the past. Using a co-design participatory approach, face and content validity of the interview guide was assessed by members of the advisory committee, including patient members, via pilot testing. This feedback was used to modify the interview guide.

One-on-one interviews were conducted with eligible participants via telephone or in person and were audio-recorded. The interviews were shaped and conducted by an experienced qualitative researcher $(\mathrm{NB})$ with expertise in managing interview dynamics, minimizing social desirability bias, and probing emergent findings in situ ${ }^{24,25}$. Interviews continued until we reached thematic saturation ${ }^{26}$.

\section{Phase II: Focus Groups}

Sample Selection and Recruitment. For subsequent focus groups, we contacted participants from Phase I that had expressed an interest in participating in Phase II, and sent recruitment letters to additional patients whose names had been provided in Phase I as needed to reach the target sample size. Eighteen patients agreed to participate; there was expected attrition with 14 attending. Of the 14 focus group participants, 11 had participated in Phase I.

Data Collection. All focus groups were facilitated by NB and AR. Discussions focused on two possible strategies to improve the patient experience with cancer screening that had emerged from Phase I interviews.

As with the interview guide, the focus group guide was developed by the research team. Face validity and content validity of the focus group guide were assessed by advisory committee members via pilot testing and the focus group guide was modified accordingly. 
Data Analysis. Data analysis took both a "bottom up" (inductive) and "top down" (deductive) qualitative approach using grounded methodology and a solution-oriented co-design lens to discover important themes and identify patient priorities ${ }^{27}$. Best practices in qualitative methodology using a grounded approach were used to record, transcribe and analyse data ${ }^{28}$. All individual interviews and focus groups were audio-taped and transcribed verbatim. Field notes were kept to collect data that cannot be captured on audiotape (e.g. dynamics, emotional aspects) ${ }^{28,29}$. Field notes helped to clarify certain points or terms used during the focus groups and to document the researchers' impressions of the research process.

The analysis of the written transcripts took an iterative, "bottom up" approach using a qualitative, descriptive content analysis technique common in qualitative research and drawing on best practices in Grounded Theory methodology 22, 23, 30. Data was also analysed from a "top down" perspective, using a health equity and solution-oriented lens, common in participatory research. Data management and analysis was facilitated using QSR International's NVivo software (version 11).

The emergent themes were expanded and clarified to create a derived model of possible strategies to improve the experience of cancer screening and encourage screening uptake for patients living with low income.

\section{RESULTS}

\section{Phase I: Individual Interviews}

Barriers to Screening. Demographic characteristics of our twenty-five interviewees are presented in Table 1. The average age was 57.8 years and the majority reported difficulty making ends meet at the end of the month. Slightly more than half were female. In interviews, study participants shared reasons why they were overdue for screening or had been overdue in the past. This barrier theme contained two main sub-themes: fear and competing priorities.

Interviewees spoke of fearing several aspects of screening:

1. The examination itself

"I think the mammogram is a very, it's very painful because your breast is very sensitive..." (P23)

"The Pap test, this one makes me feel uncomfortable and when I get nervous I get more uncomfortable, and I get tense." (P4)

Table 1 Demographic Characteristics of 25 Participants Who Completed Study Interviews

\begin{tabular}{ll}
\hline \hline Characteristic & $\boldsymbol{N ( \% )}$ \\
\hline Age, range & $32-71$ years \\
Age, average & 57.8 years \\
Gender (females) & $13(52.0)$ \\
Education & $17(68.0)$ \\
$\quad$ Completed high school, at least some post-secondary & $18(72.0)$ \\
Spoken language (English only) & $14(56.0)$ \\
Born in Canada & \\
\hline
\end{tabular}

“...our own fears defeat us you know? ...[...]... Yeah, yeah, like especially with the mammogram I mean I bet you there's still so many women that are just terrified because they envision this machine that's going to envelope their breast and, and just squash it you know?...[...]... Yeah, fear is something and that's what it, I think more than anything needs to be eliminated for screening is just get rid of the fear. Come and talk to us, get rid of the fear and the only way you are going to find this out is to come and see us." (P3)

2. The possibility of a cancer diagnosis

"I am afraid of even the name of the cancer." (P17)

"We don't want to find out the answer, it's stupid and but that's the way it works, I mean, if you told me today that if I go get screened I won't have cancer, I would go do it tomorrow... and you guys make it sound so scary..." (P12)

“....some just don't want to hear any bad news...[...]...Ignorance is bliss, if I don't know about it, I don't have to worry about it..." (P24)

"Yeah, well, why would I want to know the bad stuff? So it's like my bills at home I just throw them on the table I don't open them." (P25)

3. Triggering past trauma

"So, all this other stuff I find extraordinarily triggering. Okay, that might be fine and dandy for a person who has not been traumatized in their childhood...I can tell you right now that is the most triggering thing in the universe for someone like me. The solution is: Leave us alone. Just leave us alone.." (P19)

Patients also spoke of multiple either physical ailments or social issues to manage that were directly impacting their lives and thus served as competing priorities to staying up to date on cancer screening:

"I had other ailments, other problems..." (P16)

"Well, I mean like I said it's just with the pancreas thing. I mean I know that's there so that's my \#1 worry" (P18)

"Yeah, and trying to feed the kids, trying to keep up, keep a roof over my head, trying to go to the doctors, their health isn't at the top of their list. If you're hungry you are not, if you are hungry, you are not thinking about getting a, going to the doctor and getting tests. If I have got a choice today go to the soup kitchen here and get food or go to my doctors to get screened for cancer that I might not have, well guess what if I don't get the food I know I am going to.” (P7)

Suggestions for Improvements to Screening Outreach. When asked about approaches that the organization could use to increase screening for patients living with low income and address identified barriers, an important umbrella theme emerged, which we have called the "tea and cookies" approach: "Yeah, that's kind of what I was getting at with the old version of medicine...you sit down with her and you have tea and cookies... Because it's a more healing, yeah." 
(P19). The core element of this approach as described by participants was outreach and communication using a friendly, encouraging tone. If warnings and statistics needed to be used to encourage patients to undergo screening, participants felt they should be presented in a gentle manner. Two prevailing strategies under this approach that participants supported were the phone call strategy that was currently in use and a group education strategy.

Using a reminder phone call was seen by participants as potentially beneficial for patients who are already somewhat engaged in screening. Participants emphasized that the person making the phone call must be well trained with excellent communication skills:

"If you want to have a person feel comfortable, you are better off talking to them. Like there, it's better to hear a voice and introduce themselves so that you feel like they are a person like you are a person, they are a person." (P19)

"The tone of voice has a lot to do with it." (P7)

The idea of a group education strategy was wholly initiated by study participants and not one we had previously considered. They perceived this strategy would be desirable for patients who were not yet engaged with screening. Many participants were quite enthusiastic when they spoke of the concept of group education (although men were less likely to favour this approach) and noted the sessions should focus on health and wellness in general, not just cancer screening.

Although sending reminder letters was part of our practice's core cancer screening strategies, there was little enthusiasm for this approach among patients: " ...a lot of people get so much mail or letters, or emails nowadays it's kind of nice to have that friendly voice on the other end of the phone I think you know?" (P13)

\section{Phase II: Focus groups}

Factors to Consider for Screening Initiatives. In focus groups, participants discussed the "tea and cookies" approach of reminder phone calls and group education in further detail, highlighting important factors to consider when designing these initiatives (Table 2).

Regarding interpersonal aspects, patients valued strong trusting relationships with their providers, and wanted to feel like they had a choice in their own health journey. Patients again emphasized that past experience with trauma can trigger fear and avoidance of screening, and would be an important barrier to address. Views were mixed on if the tone used should be more authoritative versus more conversational (with men tending to favour an authoritative tone).

Regarding how screening information is presented, participants encouraged us to achieve a balance in visuals and text and in medical and plain language. They also highlighted that sufficient information must be provided about the test itself.

Participants discussed practical logistics for both initiatives that would need to be considered but there was no consensus on the time of day that group sessions should be held or on whether they should be single-gender versus mixed genders. Concerns were also raised about being able to get a hold of patients by telephone if a phone call strategy was used (Table 2).

\section{DISCUSSION}

In this qualitative, multi-phase study, patients of our primary care organization living with low income told us that a warm and encouraging outreach approach to engaging patients in cancer screening would work best to address the common barriers of fear and competing priorities and increase screening uptake. Phone calls and group education were specifically suggested as potentially promising methods, with little enthusiasm for letters. However, these views were not universal; for example, women were more likely to favour group education. These findings suggest that a multi-modal approach to cancer screening outreach is most likely to be effective, with no one solution that fits all patients, even those within the same socioeconomic group.

As a direct result of our study findings, our organization is piloting group education sessions. These sessions are focused on women overdue for at least two types of screening; in our practice, these women disproportionately live in the lowest neighbourhood income quintiles. Women are invited to attend the session by both a short, plain-language invitation letter and by telephone call. In the sessions, we have strived for the balance that patients told us they wanted: of visuals and text, and of medical and plain language, and education is on both cancer screening and prevention. The session curriculum relies heavily on one developed by our regional cancer programme's cancer screening team that has been used in many community settings. At the end of our pilot sessions, patients are given the option of having breast and cervical cancer screening immediately afterward and having faecal immunochemical test kits ordered. However, it is emphasized that undergoing screening is their choice. The sessions are led by a nurse and dietician, both of whom identify as female, to hopefully reduce the sense of power imbalance that might exist if led by a physician. Both afternoon and evening sessions are being piloted.

Our findings that patients supported group education and reminder phone calls are in line with other studies that have looked at evidence-supported cancer screening initiatives. In their meta-analysis of interventions to increase uptake of cancer screening, Stone et al found that patient reminders were among the most effective ${ }^{31}$. In their systematic review, Baron et al. found that patient reminders increase participation in breast, cervical and colorectal cancer screening; evidence was insufficient for group education ${ }^{32}$. In an Ontario study, Dunn et al. found that group education with patient navigation led to significant improvements in breast and cervical cancer screening uptake for under/never-screened women ${ }^{33}$.

Our findings that fear and competing priorities were key barriers to screening may be useful to those designing 
Table 2 Focus Group Findings on Factors to Consider for Cancer Screening Initiatives with Examples of Supporting Quotations

Theme Supporting Quotations

Interpersonal aspects

Good relationship/trust with provider

Choice in one's health journey

Consideration of patient's experience with trauma, mental illness

Tone of outreach phone calls

Presentation of information about cancer screening

Balance of visuals and text, of medical terms with lay terms

Providing enough information about test

Logistics

Daytime versus evening group sessions

Phone-specific issues for reminder phone calls

Mixing genders versus single-gender in group sessions
"Okay, if a doctor asked me to go to or a professional person, for that matter asking me to go for screening [Um hmm] I will say yes...” (P3:FG3)

"...And if you got sick chances were like when you were a kid your GP was going to come down to the house and, and check you right? So, we had like a much better closer relationship with our GPS than people do today, like I am lucky if I get 5 minutes with my GP..." (P4:FG1)

“...if you're a grown up, you ought to be able to make your own choices in life and make your own decisions and if your GP says to you know, I think it might be a nice idea if you, you know, get yourself checked, you haven't been checked in a while and you can have conversation with your GP about it right?..." (P4: FG1)

"...I am dealing with post-traumatic stress disorder, depression, anxiety, panic attacks so for me going to downtown sometimes...I'm scared, I have panic attacks, disorientated I don't know where I am, where I am going, what's going on with me [Um hmm] so, when I feel like that I am asking my neighbour, ...then I feel more comfortable [Okay] safe..." (PG2:FG3)

We are not here to be buddies just get to the point on what are you calling me for?...Yeah, just Hi, I am calling uh, Doctor's office calling for so and so...it's just get to the point, get it done, boom." (P5:FG2)

Like a robot, I don’t like that. [Yeah] Just a friendly conversation sometimes..." (P2:FG2)

"Somewhere in the middle, you know, like as opposed to using medical dictionary terms when you are talking to the average person [Um hmm], a lot of them we do know because you hear them every day... but there are terms that nobody in here understands...[...]...So, if, if you are calling from the doctor's office talk to the, talk to a plumber like he is a plumber not a, a surgeon." (P4:FG2)

"I think both, like first they say the technical way and then they say basically that means like they squish your boob in this machine and it takes a picture." (P4:FG2)

"I am not a doctor, I am not a nurse I don't know how these tests work because I've never had them so, explain it to me and that's like okay what does it mean? ..." (P4:FG2)

"I, I would ask for some more explanation, more, more detail [Um hmm], details." (P2:FG3)

"I am going to say probably evenings are best for people who are working or in school [Um hmm]. Weekends are going to be busy with family and running errands." (P:FG3)

"Not the end of the day because I am tired..." (P:FG1)

"...I am just saying if I get an, if it comes up unknown number I just don't even bother..." (P2:FG2)

"Yeah, I, I don't have an answering machine [Okay] and when somebody calls me if it's from a doctor I don't know because it's a private number, a private name." (P:FG1)

Females are dealing with one type of cancer men are dealing [Yeah] with another type of cancer

[Yeah] so..." (P6:FG2)

"I think it's very individual you know, like I don't mind to have a woman in the group..[...]...so for me it's very knowledgeable even to hear opinion from the ladies coming to the group [Um hmm] and sharing their experience right? ..." (P2:FG3) screening interventions. Practising trauma-informed care could be key to addressing fear. Trauma-informed care consists of (i) bearing witness to the patient's experience, (ii) helping patients feel they are in a safe space, (iii) actively engaging patients in their healthcare through informed choice, (iv) believing in the patient's strength and resilience and (v) incorporating processes that reflect a patient's personal and social identity ${ }^{34}$. At the very least, our findings suggest that any encounters around screening should include gently probing patients' fears if there are hesitations about screening, as well as trying to address patients' competing priorities through making screening as accessible as possible ${ }^{35,36}$.

This study has several strengths. We focused this research on patients living with low income, which is relatively unique in the literature despite this group being well established as under-screened. We also took a co-design approach to our organization's screening interventions to ensure that the patient voice was heard, and to ensure that this research had direct applicability to practice. Our co-design approach moved us away from changes to our reminder letter and brought a previously unexplored idea to the forefront. However, there are also several important limitations to note. First, if we could speak to the most difficult-to-reach patients, who would be unlikely to participate in our study, we may have received different suggestions. However, the patients we worked with verified that they were living with low income and all were, or had recently been, overdue for cancer screening. Second, we did not explore how to increase direct physician recommendation of cancer screening. Physician recommendation is considered one of the strongest and most consistent predictors of screening uptake ${ }^{37}$. However, all outreach to patients is presented as coming from their particular provider's office, and providers are encouraged to promote the group screening sessions to their eligible patients. Third, asking patients to speculate on which initiatives will increase screening uptake does not ensure that those same initiatives will be effective in the real-world setting.

We have used input from patients living with low income to co-design a new approach to cancer screening in our primary care organization, an approach that could be broadly applicable to other contexts and settings. As a result, we are now piloting a group education strategy, while still continuing to refine and explore other approaches, as we have learned from our patients that a multi-modal strategy will likely be best to maximize screening uptake. Our primary care organization will continue 
to prioritize patient input and co-design when planning quality improvement initiatives.

Acknowledgements: We would like to thank Anne Crassweiler, Sam Davie, Jean Wilson and all other members of our community advisory committee for their guidance, input and support throughout this study. $A L$ is supported by a Chair in Implementation Science at Women's College Hospital.

Corresponding Author: Aisha K. Lofters, MD PhD; MAP Centre for Urban Health Solutions, Li Ka Shing Knowledge Institute St. Michael's Hospital , 30 Bond Street, Toronto , ON M5B 1W8, Canada (e-mail: Aisha.lofters@utoronto.ca).

Funding Information This study was funded by an Academic Health Sciences Centre Alternate Funding Plan Innovation Fund from St. Michael's Hospital. AL is supported by a Canadian Institutes for Health Research (CIHR) New Investigator Award. Both AL and TK are supported as Clinician Scientists by the Departments of Family \& Community Medicine at St. Michael's Hospital and the University of Toronto. TK is also supported by CIHR and Health Quality Ontario as an Embedded Clinician Scientist and as the Fidani Endowed Chair in Improvement and Innovation at the University of Toronto.

\section{Compliance with Ethical Standards:}

Ethics approval for the study was provided by the St. Michael's Hospital Research Ethics Board.

Conflict Of Interest: The authors declare that they do not have a conflict of interest.

\section{REFERENCES}

1. Cancer Screening in Canada: An Overview of Screening Participation for Breast, Cervical and Colorectal Cancer. Toronto: Canadian Partnership Against Cancer; 2015.

2. Cervical Screening - CCO: Cancer Care Ontario; 2012 [Available from: https://www.cancercare.on.ca/pcs/screening/cervscreening/.

3. About the Ontario Breast Screening Program: Cancer Care Ontario; [February 9, 2016]. Available from: https://www.cancercare.on.ca/pcs/ screening/breastscreening/OBSP/.

4. CancerCareOntario. Screening - CCO [Available from: https://www. cancercare.on.ca/pcs/screening/.

5. Colorectal Cancer Screening Participation: Cancer Quality Council of Ontario; [Available from: http://www.csqi.on.ca/by_patient_journey/ screening/colorectal_screening_participation/.

6. Screening: Cancer Screening Quality Index; [Available from: http://www. csqi.on.ca/by_patient_journey/screening/.

7. Elit L, Krzyzanowska M, Saskin R, Barbera L, Razzaq A, Lofters A, et al. Sociodemographic factors associated with cervical cancer screening and follow-up of abnormal results. Can Fam Physician. 2012;58(1):e22-31.

8. Fernandes $\mathbf{K}$, Sutradhar R, Borkhoff C, Baxter N, Lofters A, Rabeneck $\mathbf{L}$, et al. Cross-sectional small area variation study of cancer, glucose, and cholesterol screening in Ontario: data to motivate community and primary care strategies to improve participation. CMAJ Open.

9. Lofters AK, Moineddin R, Hwang Sw, Glazier RH. Predictors of low cervical cancer screening among immigrant women in Ontario, Canada. BMC Womens Health. 2011;11:20.

10. Lofters AK, Ng R, Lobb $\mathbf{R}$. Primary care physician characteristics associated with cancer screening: a retrospective cohort study in Ontario, Canada. Cancer Med. 2014.

11. Shen SC, Lofters A, Tinmouth J, Paszat L, Rabeneck L, Glazier RH. Predictors of non-adherence to colorectal cancer screening among immigrants to Ontario, Canada: a population-based study. Prev Med. 2018;111:180-9.

12. Borkhoff CM, Saskin R, Rabeneck L, Baxter NN, Liu Y, Tinmouth J, et al. Disparities in receipt of screening tests for cancer, diabetes and high cholesterol in Ontario, Canada: a population-based study using areabased methods. Can J Public Health. 2013;104(4):e284-90.

13. Income and Health: Opportunities to achieve health equity in Ontario. Toronto: Health Quality Ontario; 2016.
14. Vahabi M, Lofters A, Kumar M, Glazier RH. Breast cancer screening disparities among urban immigrants: a population-based study in Ontario, Canada. BMC Public Health. 2015;15:679.

15. Lofters AK, Schuler A, Slater M, Baxter NN, Persaud N, Pinto AD, et al. Using self-reported data on the social determinants of health in primary care to identify cancer screening disparities: opportunities and challenges. BMC Fam Pract. 2017;18(1):31.

16. Feldman J, Davie S, Kiran T. Measuring and improving cervical, breast, and colorectal cancer screening rates in a multi-site urban practice in Toronto, Canada. BMJ Qual Improv Rep. 2017;6(1).

17. Low Income Cut-Offs: Statistics Canada; [Available from: http://www. statcan.gc.ca/pub/75f0002m/2012002/lico-sfr-eng.htm.

18. Kiran T, Davie S, Moineddin R, Lofters A. Mailed Letter Versus Phone Call to Increase Uptake of Cancer Screening: A Pragmatic, Randomized Trial. J Am Board Fam Med. 2018;31(6):857-68.

19. Bate P, Robert G. Experience-based design: from redesigning the system around the patient to co-designing services with the patient. Qual Saf Health Care. 2006;15(5):307-10.

20. Trischler J, Pervan SJ, Kelly SJ, Scott DR. The value of codesign: The effect of customer involvement in service design teams. Journal of Service Research. 2017;21(1):75-100.

21. Israel BA, Schulz AJ, Parker EA, Becker AB, Health C-CPf. Community-based participatory research: policy recommendations for promoting a partnership approach in health research. Educ Health (Abingdon). 2001;14(2): 182-97.

22. Sandelowski M. Whatever Happened to Qualitative Description? Research in Nursing \& Health. 2000;23:334-40.

23. Strauss A, Corbin J. Basics of Qualitative Research - Techniques and Procedures for Developing Grounded Theory. London: Sage Publications; 1998.

24. Creswell JW. Qualitative Inquiry and Research Design: Choosing Among Five Traditions. Thousand Oaks: Sage Publications; 1998.

25. Bauer MW, Gaskell G. Qualitative researching with text, image and sound: A practical handbook. London: Sage Publications; 2000.

26. Kvale S. InterViews: An Introduction to Qualitative Research Interviewing. Thousand Oaks: Sage Publications; 1996.

27. Braun V, Clarke V. Using thematic analysis in psychology. Qualitative research in psychology. 2006;3:77-101.

28. Novick G. Is there a bias against telephone interviews in qualitative research? Res Nurs Health. 2008;31(4):391-8.

29. Tessier S. From Field Notes, to Transcripts, to Tape Recordings: Evolution or Combination? International Journal of Qualitative Methods. 2012;11(4):446-60.

30. Morse JM. The significance of saturation. Qualitative Health Research. 1995;5:147-9.

31. Stone EG, Morton SC, Hulscher ME, Maglione MA, Roth EA, Grimshaw JM, et al. Interventions that increase use of adult immunization and cancer screening services: a meta-analysis. Ann Intern Med. 2002; 136(9):641-51.

32. Baron RC, Rimer BK, Breslow RA, Coates RJ, Kerner J, Melillo S, et al. Client-directed interventions to increase community demand for breast, cervical, and colorectal cancer screening a systematic review. Am J Prev Med. 2008;35(1 Suppl):S34-55.

33. Dunn SF, Lofters AK, Ginsburg OM, Meaney CA, Ahmad F, Moravac MC, et al. Cervical and Breast Cancer Screening After CARES: A Community Program for Immigrant and Marginalized Women. Am J Prev Med. 2017;52(5):589-97.

34. Purkey E, Patel R, Phillips SP. Trauma-informed care: Better care for everyone. Can Fam Physician. 2018;64(3):170-2.

35. Hall N, Birt L, Rees CJ, Walter FM, Elliot S, Ritchie M, et al. Concerns, perceived need and competing priorities: a qualitative exploration of decision-making and non-participation in a population-based flexible sigmoidoscopy screening programme to prevent colorectal cancer. BMJ Open. 2016;6(11):e012304.

36. Kouyoumdjian FG, McConnon A, Herrington ERS, Fung $\mathbf{K}$, Lofters A, Hwang SW. Cervical Cancer Screening Access for Women Who Experience Imprisonment in Ontario, Canada. JAMA Netw Open. 2018;1(8):e185637.

37. Hudson SV, Ferrante JM, Ohman-Strickland P, Hahn KA, Shaw EK, Hemler $\mathbf{J}$, et al. Physician recommendation and patient adherence for colorectal cancer screening. J Am Board Fam Med. 2012;25(6):782-91.

Publisher's Note Springer Nature remains neutral with regard to jurisdictional claims in published maps and institutional affiliations. 